\title{
Phase Diagram and Entanglement of Ising Model With Dzyaloshinskii-Moriya Interaction (IDM)
}

\author{
R. Jafari, ${ }^{1,2}$ M. Kargarian, ${ }^{3}$ A. Langari, ${ }^{3}$ and M. Siahatgar ${ }^{3}$ \\ ${ }^{1}$ Institute for Advanced Studies in Basic Sciences, Zanjan 45195-1159, Iran \\ ${ }^{2}$ School of physics, IPM (Institute for Studies in Theoretical Physics and Mathematics), \\ P. O. Box: 19395-5531, Tehran, Iran \\ ${ }^{3}$ Physics Department, Sharif University of Technology, Tehran 11155-9161, Iran
}

(Dated: June 19, 2018)

\begin{abstract}
We have studied the phase diagram and entanglement of the one dimensional Ising model with Dzyaloshinskii-Moriya (DM) interaction. We have applied the quantum renormalization group (QRG) approach to get the stable fixed points, critical point and the scaling of coupling constants. This model has two phases, antiferromagnetic and saturated chiral ones. We have shown that the staggered magnetization is the order parameter of the system and DM interaction produces the chiral order in both phases. We have also implemented the exact diagonalization (Lanczos) method to calculate the static structure factors. The divergence of structure factor at the ordering momentum as the size of systems goes to infinity defines the critical point of the model. Moreover, we have analyzed the relevance of the entanglement in the model which allows us to shed insight on how the critical point is touched as the size of the system becomes large. Nonanalytic behavior of entanglement and finite size scaling have been analyzed which is tightly connected to the critical properties of the model. It is also suggested that a spin-fluid phase has a chiral order in terms of new spin operators which are defined by a nonlocal transformation.

PACS numbers: 75.10.Pq, 73.43.Nq, 03.67.Mn, 64.60.ae
\end{abstract}

\section{INTRODUCTION}

At zero temperature, the properties of quantum manybody system is dictated by the structure of its ground state. The degree of complexity of this structure is different for various systems. It ranges from exceptionally simple case (when a strong magnetic field aligns all the spins of a ferromagnet along the field direction, producing a product or unentangled state) to more intricate situation where entanglement pervades the ground state of system. Thus, entanglement appears naturally in low temperature quantum many body systems, and it is at the core of relevant quantum phenomena, such as superconductivity ${ }^{\underline{1}}$, quantum Hall effect ${ }^{2}$, and other quantum phase transitions ${ }^{3}$. Quantum phase transitions have been one of the most interesting topics of strongly correlated systems during the last decade. It is basically a phase transition at zero temperature where the quantum fluctuations play the dominant role ${ }^{4}$. Suppression of the thermal fluctuations at zero temperature introduces the ground state as the representative of the system. The properties of the ground state may be changed drastically shown as a non-analytic behavior of a physical quantity by reaching the quantum critical point (QCP). This can be obtained by tunning a parameter in the Hamiltonian, such as the magnetic field or the amount of disorder. The ground state of a typical quantum many body system consists of a superposition of a huge number of product states. Understanding this structure is equivalent to establishing how subsystems are interrelated, which in turn is what determines many of the relevant properties of the system. In this sense, the study of entanglement offers an attractive theoretical framework from which one may be able to go beyond customary approaches to the physics of quantum collective phenomena ${ }^{5}$.

Recently some novel magnetic properties were discovered in a variety of quasi-one dimensional materials that are known to belong to the class of DzyaloshinskiiMoriya $(\mathrm{DM})\left(\vec{D} \cdot\left(\overrightarrow{S_{i}} \times \overrightarrow{S_{j}}\right)\right)$ magnet to explain helical magnetic structures. The relevance of antisymmetric superexchange interactions in spin Hamiltonians which describe quantum antiferromagnetic (AF) systems was introduced phenomenologically by Dzyaloshinskii ${ }^{6}$. Moriya showed later, that such interactions arises naturally in the perturbation theory due to the spin-orbit coupling in magnetic systems with low symmetry ${ }^{7}$. Some AF systems are expected to be described by DM interaction, such as $\mathrm{Cu}\left(\mathrm{C}_{6} \mathrm{D}_{5} \mathrm{COO}\right)_{2} 3 \mathrm{D}_{2} \mathrm{O}^{8,9}, \mathrm{Yb}_{4} A s_{3}{ }^{10,11,12}$, $\mathrm{BaCu}_{2} \mathrm{Si}_{2} \mathrm{O}_{7} \stackrel{13}{ }, \alpha-\mathrm{Fe}_{2} \mathrm{O}_{3}, \mathrm{LaMnO}_{3} \underline{14}$ and $\mathrm{K}_{2} \mathrm{~V}_{3} \mathrm{O}_{8}{ }^{15}$, exhibit unusual and interesting magnetic properties due to quantum fluctuations and/or in the presence of an applied magnetic field ${ }^{14,16,17} . \quad \mathrm{La}_{2} \mathrm{CuO}$ also belongs to the class of DM antiferromagnets, which is a parent compound of high-temperature superconductors 18 . This has stimulated extensive investigations of the properties which are created from DM interaction. This interaction is however, rather difficult to handle analytically, which makes the interpretation of experimental data to be hard. In addition, more knowledge in this respect expand our understanding of many interesting quantum phenomena of low-dimensional magnetic materials.

Recent discovery of an unusual strong coupling between the ferroelectric (FE) and magnetic order parameters has also revived the interest in the magnetoelectric effect $1 \frac{19}{}$. Due to the possibility of easily controlling the electrical properties using magnetic field, search of 


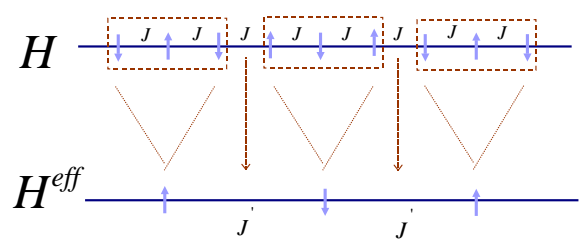

FIG. 1: (color online)The decomposition of chain into three site blocks Hamiltonian $\left(H^{B}\right)$ and inter-block Hamiltonian $\left(H^{B B}\right)$.

compounds, in which the magnetic order is incommensurate with lattice period, is of particular interest for future applications 20.21 . Generally, certain types of magnetic order can lower the symmetry of the system to that of the polar groups, which allow for ferroelectricity. According to the recent experimental results, helical magnetic structure are the most likely candidates to host ferroelectericity $22,23,24$. It has been shown that the DM interaction induces a $\mathrm{FE}$ lattice displacement and helps to stabilize helical magnetic structures at low temperature ${ }^{25}$.

In the present paper, we have studied a one dimensional AF Ising model with DM interaction using the quantum renormalization group (QRG) and numerical exact diagonalization methods. In the next section the QRG approach will be explained and the renormalization of coupling constants are obtained. In section III, we will obtain the phase diagram, fixed points, critical points and the staggered magnetization as the order parameter of the model. We will also introduce the chiral order as an ordering which is created by DM interaction. The exponent which shows the divergence of correlation function close to the critical point $(\nu)$, the dynamical exponent $(z)$ and the exponent which shows the vanishing of staggered magnetization near the critical point $(\beta)$ will be also calculated. We then present the numerical exact diagonalization results on finite sizes of $N=12,16,20$ and 24. In section IV we will calculate the renormalization of entanglement 26.27 for this model and we will show that it has a scaling behavior near the QCP which is directly related to critical properties of the model.

\section{QUANTUM RENORMALIZATION GROUP}

The main idea of the RG method is the mode elimination or the thinning of the degrees of freedom followed by an iteration which reduces the number of variables step by step until a more managable situation is reached. We have implemented the Kadanoff's block approach to do this purpose, because it is well suited to perform analytical calculations in the lattice models and they are conceptually easy to be extended to the higher dimensions $28,29,30,31$. In the Kadanoff's method, the lattice is divided into blocks in which the Hamiltonian is exactly diagonalized. By selecting a number of low-lying eigenstates of the blocks the full Hamiltonian is projected onto these eigenstates which gives the effective (renormalized) Hamiltonian.

The Hamiltonian of Ising model with DM interaction in the $z$ direction on a periodic chain of $N$ sites is

$$
H=\frac{J}{4}\left[\sum_{i=1}^{N} \sigma_{i}^{z} \sigma_{i+1}^{z}+D\left(\sigma_{i}^{x} \sigma_{i+1}^{y}-\sigma_{i}^{y} \sigma_{i+1}^{x}\right)\right]
$$

The effective Hamiltonian (in the first order renormalization group prescription) is

$$
\begin{gathered}
H^{\text {eff }}=H_{0}^{\text {eff }}+H_{1}^{e f f}, \\
H_{0}^{\text {eff }}=P_{0} H^{B} P_{0} \quad, \quad H_{1}^{e f f}=P_{0} H^{B B} P_{0} .
\end{gathered}
$$

We have considered a three site block procedure defined in Fig.(1). The block Hamiltonian $\left(H_{B}=\sum h_{I}^{B}\right)$ of the three sites and its eigenstates and eigenvalues are given in Appendix A. The three site block Hamiltonian has four doubly degenerate eigenvalues (see appendix A). $P_{0}$ is the projection operator of the ground state subspace which is defined by $\left(P_{0}=|\Uparrow\rangle\left\langle\psi_{0}|+| \Downarrow\right\rangle\left\langle\psi_{0}^{\prime}\right|\right)$, where $\left|\psi_{0}\right\rangle$ and $\left|\psi_{0}^{\prime}\right\rangle$ are the doubly degenerate ground states, $|\Uparrow\rangle$ and $|\Downarrow\rangle$ are the renamed base kets in the effective Hilbert space. We have kept two states $\left(\left|\psi_{0}\right\rangle\right.$ and $\left.\left|\psi_{0}^{\prime}\right\rangle\right)$ for each block to define the effective (new) sites. Thus, the effective site can be considered as a spin $1 / 2$. The effective Hamiltonian is not exactly similar to the initial one, i.e, the sign of DM interaction is changed

$$
H^{e f f}=\frac{J^{\prime}}{4}\left[\sum_{i=1}^{N} \sigma_{i}^{z} \sigma_{i+1}^{z}-D^{\prime}\left(\sigma_{i}^{x} \sigma_{i+1}^{y}-\sigma_{i}^{y} \sigma_{i+1}^{x}\right)\right],
$$

where $J^{\prime}$ and $D^{\prime}$ are the renormalized coupling constants. To have a self-similar Hamiltonian, we implement a $\pi$ rotation around $x$ axis on all sites $\left(\sigma_{i}^{z} \rightarrow-\sigma_{i}^{z}, \sigma_{i}^{y} \rightarrow-\sigma_{i}^{y}\right)$. We note to interpret our final results in terms of this transformation. The renormalized coupling constants are functions of the original ones which are given by the following equations.

$J^{\prime}=J\left(\frac{1+q}{2 q}\right)^{2} \quad, \quad D^{\prime}=\frac{16 D^{3}}{(1+q)^{2}} \quad, \quad q=\sqrt{1+8 D^{2}}$.

We will implement this approach in the next sections to obtain the phase diagram and entanglement properties of the model.

\section{PHASE DIAGRAM}

The RG equations show the scaling of $J$ coupling to zero which represents the renormalization of energy scale. At zero temperature, a phase transition occurs upon variation of the parameters in the Hamiltonian. In the absence of DM interaction $(D=0)$ the ground state of the Ising model is the Neel ordered state. However, for 
$D \neq 0$ the DM interaction makes a tendency for spins to be oriented in the $X Y$ plane. A nonzero value of $D$ increases the fluctuations which destroys the AF ordering in $z$ direction at some finite value of $D=D_{c}$. Simultaneously, the chiral order grows up and will saturate as $D \rightarrow \infty$. The quantum phase transition can be interpreted as the antiferromagnet to saturated chiral (SC) order transition at $D=D_{c}$. The RG flow shows that in the AF phase $\left(D<D_{c}=1\right)$, the DM coupling $(D)$ goes to zero and in the SC phase $D$ goes to infinity (Fig.(2)). We have probed the AF-SC transition by calculating the staggered magnetization $S_{M}$ (See appendix B) in the $z$ direction as an order parameter (Fig.(3)),

$$
S_{M}=\frac{1}{N} \sum_{i=1}^{N} \frac{(-1)^{i}}{2}\left\langle\sigma_{i}^{z}\right\rangle
$$

$S_{M}$ is zero in the SC phase and has a nonzero value in the AF phase. Thus, the staggered magnetization is the proper order parameter to represent the AF-SC transition. We have plotted $S_{M}$ versus $D$ in Fig.(3). It has its maximum value at $D=0$ and continuously decreases with increase of $D$ to zero at $D=1$. Moreover, we have calculated the chiral order ${ }^{32}\left(C_{h}\right)$ in the $z$ direction (See appendix B) which increases with $D$ and saturates for $D \rightarrow \infty$ (Fig 3),

$$
C_{h}=\frac{1}{N} \sum_{i=1}^{N} \frac{1}{4}\left\langle\left(\sigma_{i}^{x} \sigma_{i+1}^{y}-\sigma_{i}^{y} \sigma_{i+1}^{x}\right)\right\rangle .
$$

The chiral order has a nonzero value in both $\mathrm{AF}$ and SC phases which can not be a proper order parameter to distinguish the quantum phase transition. However, it shows that the onset of DM interaction sets up the chiral order immediately. A classical picture of the chiral order in terms of the spin projection on the $x y$-plane has been plotted in Fig 4

We have also calculated the critical exponents at the critical point $(D=1)$. In this respect, we have obtained the dynamical exponent, the exponent of order parameter and the diverging exponent of the correlation length. This corresponds to reaching the critical point from the AF phase by approaching $D \rightarrow 1$. The dynamical exponent is given by $z=\left[\ln \left(J / J^{\prime}\right)_{D=1}\right] /\left[\ln \left(n_{B}\right)\right] \simeq 0.73$, where $n_{B}=3$ is the number of sites in each block. The staggered magnetization close to the quntum critical point goes to zero like $S_{M} \sim|D-1|^{\beta}$ where $\beta \simeq 1.15$ and is obtained by $\beta=\left[\ln \left(S_{M}^{\prime} / S_{M}\right)\right] /\left.\ln \left[\frac{d D^{\prime}(D)}{d D}\right]\right|_{D=1}$ where prime denotes the renormalized quantity. The correlation length diverges $\xi \sim|D-1|^{-\nu}$ with exponent $\nu \simeq 2.15$ which is expressed by $\nu=\left[\ln \left(n_{B}\right)\right] /\left.\ln \left[\frac{d D^{\prime}(D)}{d D}\right]\right|_{D=1}$. The detail of this calculation is similar to what has been presented in Ref.[28].

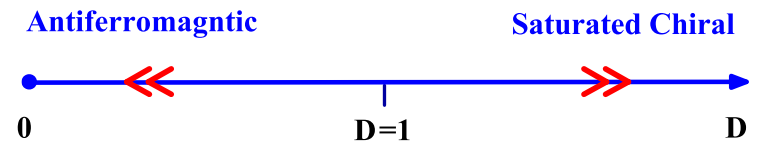

FIG. 2: (color online) Phase diagram of the Ising model with DM interaction. Arrows show the running of coupling constant under RG iteration.

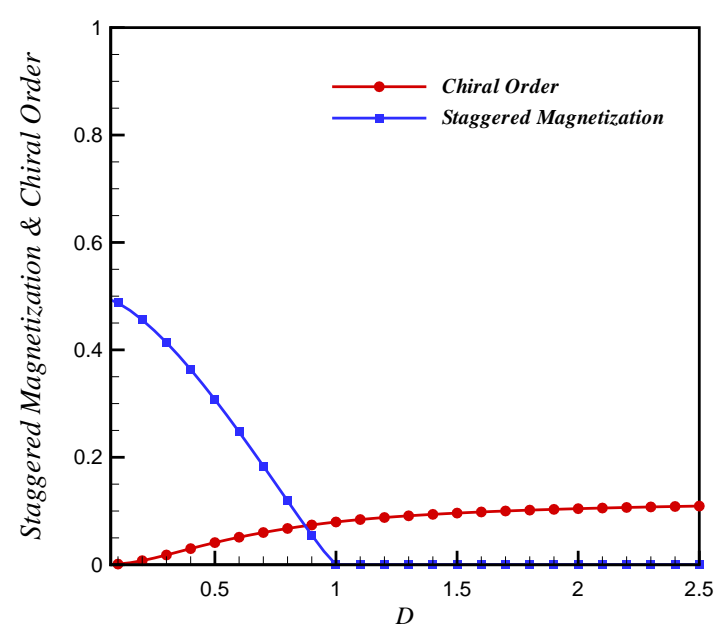

FIG. 3: (color online) Chiral order (filled circles) and Staggered Magnetization (filled squares) versus D.

\section{NUMERICAL RESULTS}

We have implemented the exact diagonalization method based on Lanczos algorithm to get the ground state properties of the Hamiltonian defined in Eq.(11). The Hamiltonian does not commute with $S^{z}=$ $(1 / 2) \sum_{i} \sigma_{i}^{z}$, which imposes to consider the full Hilbert space for computations. We have considered a periodic chain of length $N=8,12,16,20$ and 24 in our calculations. We have first calculated the ground state energy for different sizes. We have observed the size dependence for ground state energy is weak which make us to extrapolate our results to get the ground state energy per site $\left(E_{0} / N\right)$ as its thermodynamic limit $(N \rightarrow \infty)$. We

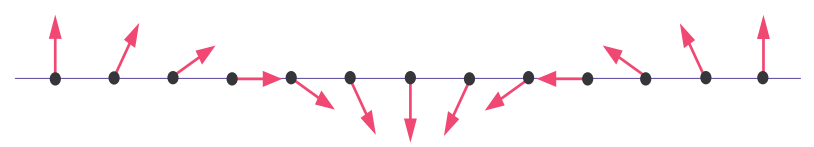

FIG. 4: (color online) A classical picture of spin orientation in the $x y$ plain where the angle between neibouring spins depend on the D value (see appendix C). 


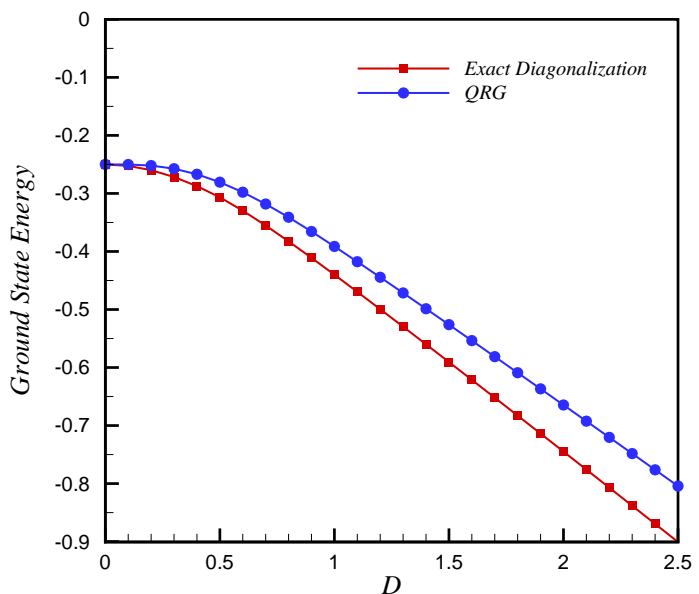

FIG. 5: (color online) The ground state energy versus $D$. The result of QRG (filled circle) is compared with the extrapolated values of exact diagonalization (filled squares).

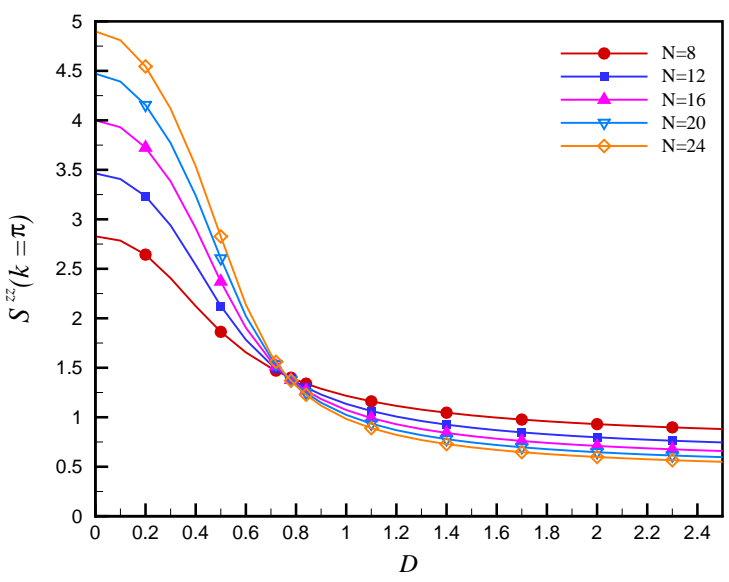

FIG. 6: (color online) The z-component static structure factor at antiferromagnetic ordering vector $k=\pi$ versus D. A more clear picture about the crossing point is plotted in Fig.(7).

have plotted $E_{0} / N$ versus $D$ in Fig.(15). Our results show that the QRG result for $E_{0}$ is close to the exact diagonalization one which justifies the correct trend versus $D$ although the values have around 10 percent error. It is a good evidence that the QRG result is reliable at least to get the qualitative picture of the model.

Since the numerics is done on finite size systems the symmetry breaking can not occur in our calculation to show the nonzero value of the order parameter. Instead, the structure factor shows a divergent bahaviour at ordering momentum by increasing the size of system. The structure factor at momentum $k$ is defined by the follow-

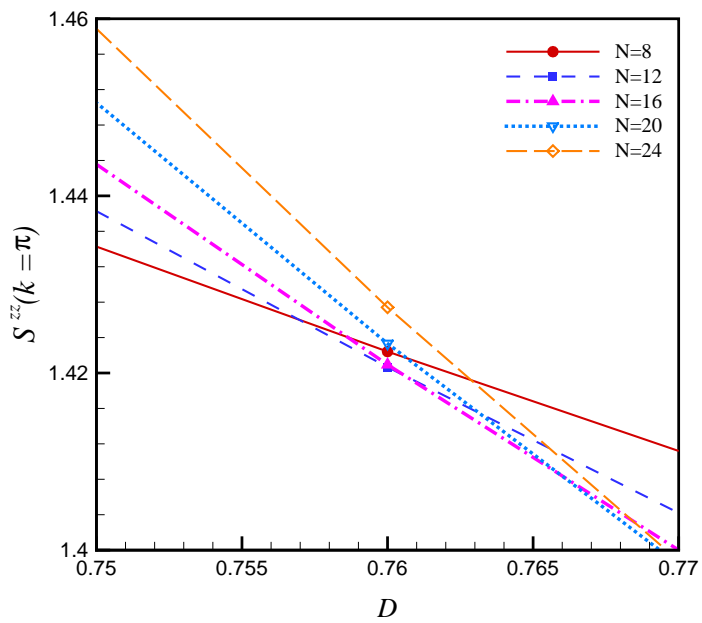

FIG. 7: (color online) A closer look at the crossing point of Fig.(6) which shows that different plots do not cross each other at a single point. The crossing point of two successive size occurs at larger value of D upon increasing size.

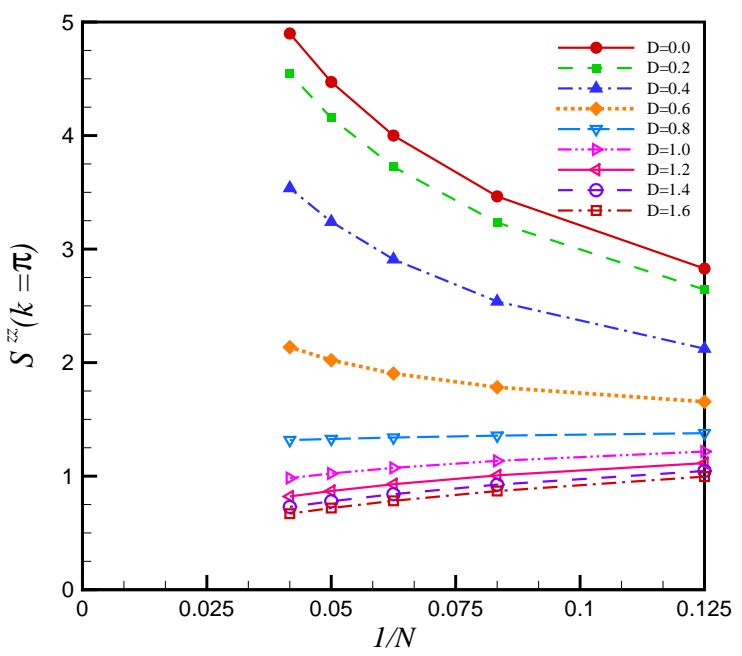

FIG. 8: (color online) The z-component structure factor for different $D$ versus $1 / N$. Plots for $D \lesssim 0.8$ show diverging behaviour as $N \rightarrow \infty$ while those for $D>0.8$ becomes finite in the thermodynamic limit.

ing relation

$$
S^{\alpha \alpha}(k)=\frac{1}{\sqrt{N}} \sum_{r=0}^{N-1}\left\langle\sigma_{i}^{\alpha} \sigma_{i+r}^{\alpha}\right\rangle e^{i k r} .
$$

The $z$-component of structure factor $\left(S^{z z}(k)\right)$ versus $k$ has a sharp peak at $k=\pi$ for $D \lesssim 0.8$ representing the antiferromagnetic order. To justify if the peak corresponds to the true long range order (LRO) or it is just 


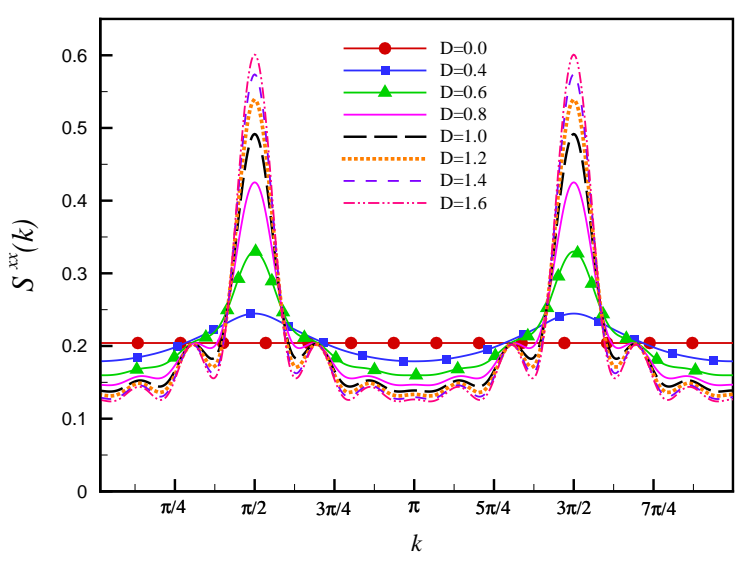

FIG. 9: (color online) The x-component structure factor versus momentum $(k)$ for different $D$.

a local order we have computed the structure factor for different size of chains $(N)$. We have plotted in Fig.(6) $S^{z z}(k=\pi)$ versus $D$ for different chain lengths $(N)$. The peak height increases for $D \lesssim 0.8$ and decreases for $D>0.8$. To have a clear picture of these data, we have plotted in Fig.(8) the same data for fixed $D$ value versus $1 / N$. We observe a divergent behaviour for the $z$-component structure factor for $D \lesssim 0.8$ and diminishing for $D>0.8$. This justifies antiferromagnetic LRO for $D \lesssim 0.8$. Although the plots for different $N$ in Fig.(6) show to cross each other at a single point $D^{*}=0.76$ the fine tunning data close to this point (Fig.(7)) represent different crossings for two successive $N$ values. It is the manifestation of finite size scaling which exist in our numerics. Therefore, the true critical point $\left(D_{c}\right)$ which should be the case for $N \rightarrow \infty$ is greater than $D^{*}=0.76$. The investigation which shows the relation between the Ising model and DM interaction with the anisotropic Heisenberg model (XXZ) verifies that the critical point should be at $D_{c}=1$. It will be discussed in Sec VI.

To get a picture on the type of ordering in the $x y$ plane we have plotted the $x$ structure factor of $N=24$ for different $D$ values versus $k$ in Fig.(9). Due to symmetry we have $S^{x x}(k)=S^{y y}(k)$, thus, we only present data for $S^{x x}(k)$. The $x$-component structure factor show two strong peaks at $k=\pi / 2,3 \pi / 2$. It is a justification of spiral order in the $x y$ plane. However, this is a local ordering and is not a true LRO. We have also plotted in Fig.(10) the value of $S^{x x}(k=\pi / 2)$ versus $1 / N$ for different $D$ values. All data in Fig.(10) show diminishing behaviour as $N \rightarrow \infty$. This justifies that the spiral (chiral) order which exist in the $x y$ plane for $D \neq 0$ is not a true LRO.

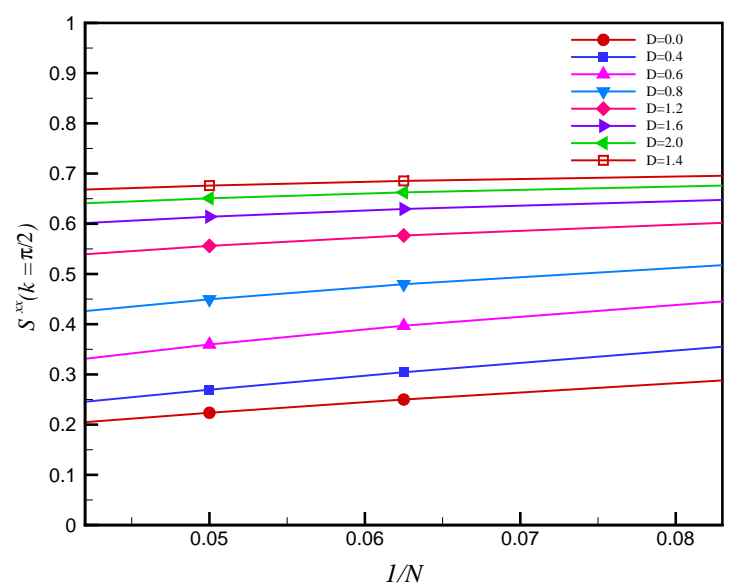

FIG. 10: (color online) The finite size scaling of $S^{x x}(k=\pi / 2)$. All plot show non-divergent behaviour as $N \rightarrow \infty$ representing no long range order but a local order.

\section{ENTANGLEMENT AND ITS SCALING PROPERTY}

In this section we calculate the entanglement of the model using the idea of renormalization group 26 . As we have mentioned previously, a finite size block is treated exactly to calculate the physical quantities. The coupling constants of a finite size blocks are renormalized via the QRG prescription to give the large size behavior. Bipartite entanglement, i.e the entanglement between some degrees of freedom and the rest of system, is quantified by von-Neumann entropy of eigenvalues of the reduced density matrix. In our case, we first calculate the entropy of the middle site and the remaining sites of a single block (see Fig.1). The entanglement is easily calculated, since the density matrix is defined by

$$
\varrho=\left|\psi_{0}\right\rangle\left\langle\psi_{0}\right|,
$$

where $\left|\psi_{0}\right\rangle$ has been introduced in Appendix (Eq. (A2)). The results will be the same if we consider $\left|\psi_{0}^{\prime}\right\rangle$ to construct the density matrix.

The density matrix defined in Eq.(4) is traced over sites 1 and 3 to get the reduced density matrix for site 2 $\left(\varrho_{2}\right)$ which gives

$$
\varrho_{2}=\frac{1}{2 q(1+q)}\left(\begin{array}{cc}
8 D^{2} & 0 \\
0 & (1+q)^{2}
\end{array}\right) .
$$

The von-Neumann entropy is then

$$
\begin{aligned}
E= & -\frac{8 D^{2}}{2 q(1+q)} \log _{2} \frac{8 D^{2}}{2 q(1+q)} \\
& -\frac{(1+q)^{2}}{2 q(1+q)} \log _{2} \frac{(1+q)^{2}}{2 q(1+q)} .
\end{aligned}
$$




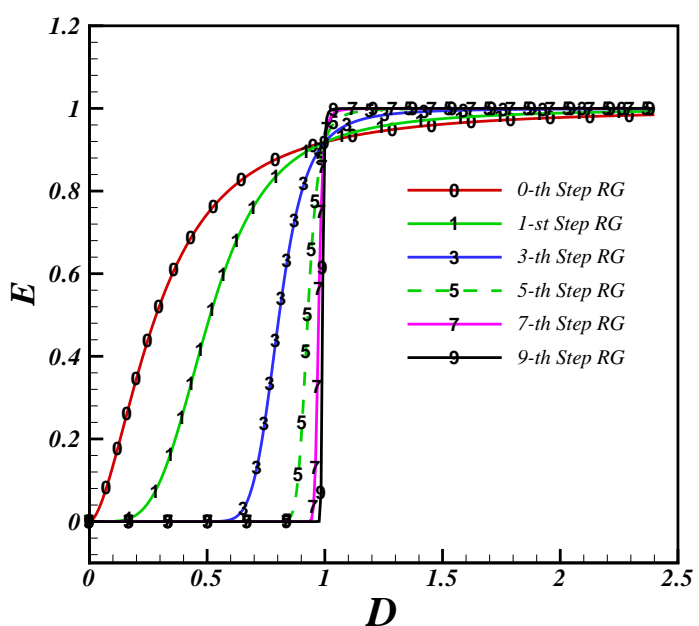

FIG. 11: (color online) Representation of the evolution of entanglement entropy in terms of RG iterations.

In the spirit of $\mathrm{RG}$, the first iteration of RG represents a chain of $3^{2}$ sites which is described effectively by three effective sites interacting via the renormalized coupling constants. Having this in mind, we understand that in the first RG iteration the von-Neumann entropy with renormalized coupling constant yields the entanglement between effective degrees of freedom. The variation of entanglement $(E)$ versus $D$ is plotted in Fig 11 . Different plots show the evolution of $E$ under QRG iterations. In other words, the different iteration of QRG show how the entanglement evolves as the size of chain is increased. Long wavelength behaviors are captured as the RG iterations are increased. In Fig 11 we see that in the gapped phase, i.e AF, and the long-wavelength limit the entanglement is suppressed while in the SC phase the entanglement gets maximum value due to the DM interaction in the $X Y$ plane that induces a state with strong quantum correlation. Such a behavior has also be seen in the $X X Z$ model 27 .

A common feature of the second order phase transitions is the appearance of nonanalytic behavior in some physical quantities or their derivatives as the critical point is crossed 33 . It is also accompanied by a scaling behavior since the correlation length diverges and there is no characteristic length scale in the system at the critical point. Entanglement as a direct measure of quantum correlations indicates the critical behavior such as diverging of its derivative as the phase transition is crossed 34 . It has been verified that the entanglement in the vicinity of critical point of Ising model in transverse field (ITF) and $X X$ model in transverse field shows a scaling behavior. Investigating the nonanaliticity, e.g a divergence, and finite size scaling provides excellent estimates for the quantum critical point. A precise connection between the entanglement in quantum information

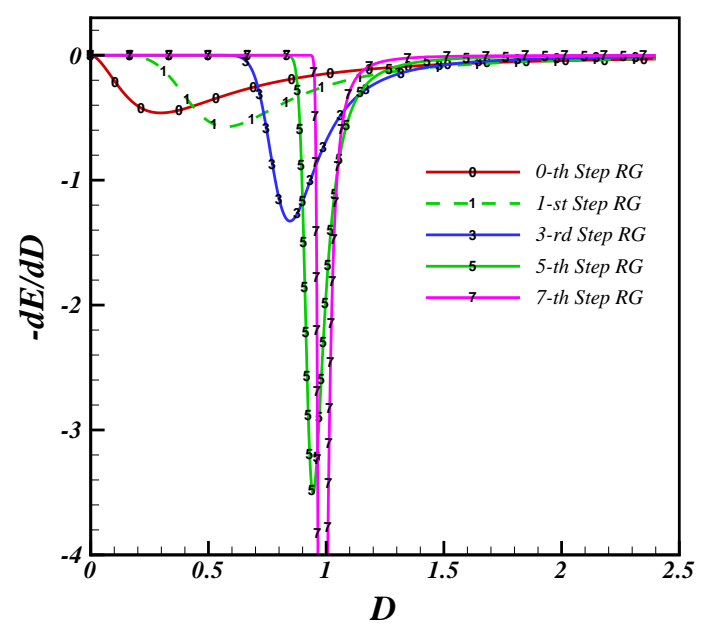

FIG. 12: (color online) First derivative of entanglement entropy and its manifestation towards divergence as the number of RG iterations increases (Fig 11).

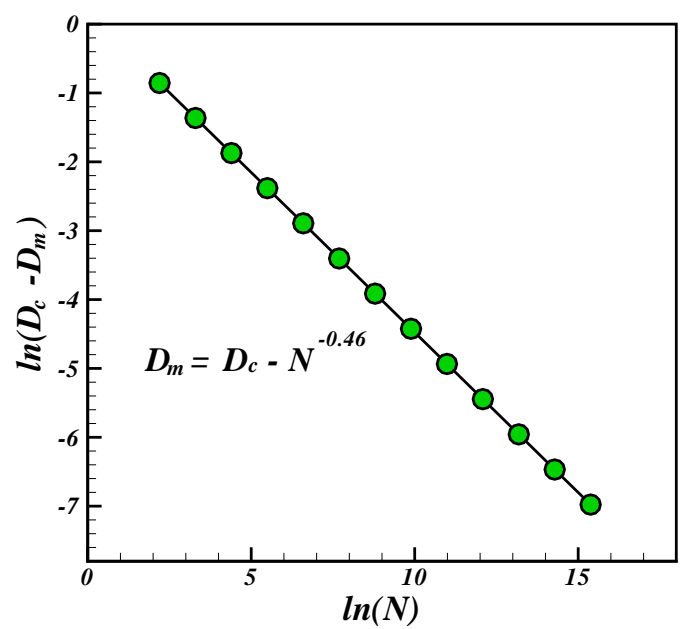

FIG. 13: (color online) The scaling behavior of $D_{m}$ in terms of system size $(N)$ where $D_{m}$ is the position of minimum in Fig 12

theory and the critical phenomena in condensed matter physics has been established ${ }^{35}$, where the scaling properties of the entanglement in spin chain systems, both near and at a quantum critical point have been investigated. The first derivative of entanglement let us to get more insight on the qualitative variation of the ground state as the critical point is touched. To this end we have calculated the first derivative of entanglement which has been depicted in Fig 12. Such a computation determines the scaling law of entanglement in one-dimensional spin sys- 


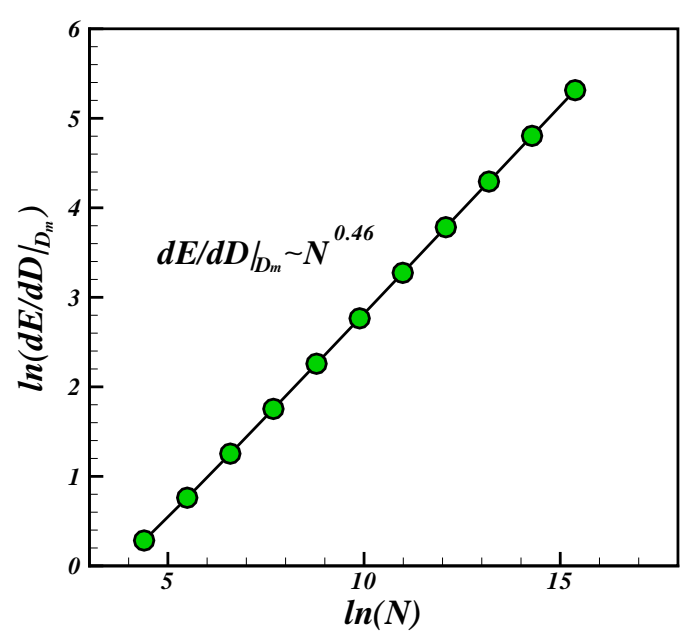

FIG. 14: (color online) The logarithm of the absolute value of minimum, $\ln \left(d E /\left.d D\right|_{D_{m}}\right)$, versus the logarithm of chain size, $\ln (N)$, which is linear and shows a scaling behavior. Each point corresponds to the minimum value of a single plot of Fig 12

tems, while explicitly uncovering an accurate correspondence with the critical properties of the model. As the size of system becomes large through RG iterations, the derivative of entanglement tends to diverge close to the critical point. All plots in Fig 12 with respect to the critical point have an asymmetrical shape. Each plot reveals a minimum in the gapped phase, i.e $\mathrm{AF}$ for $0 \leq D<1$, the minimum becomes more pronounced close to the critical point, $D=1$. It manifests that the ground state of the gapped phase of the model undergoes a strong qualitative change when approaching the quantum critical point while the corresponding change in the SC phase is rather small. A similar situation has also been observed in the $X X Z$ mode ${ }^{27}$. This behavior is comparable with results on ITF model where the system in both sides of the critical point is gapfull, so the derivative of the entanglement tends to diverge symmetrically ${ }^{26}$.

More information can be obtained when the minimum values of each plot and their positions are analyzed. The position of the minimum $\left(D_{m}\right)$ of $\frac{d E}{d D}$ tends towards the critical point like $D_{m}=D_{c}-N^{-0.46}$ which has been plotted in Fig 13. Moreover, we have derived the scaling behavior of $y \equiv\left|\frac{d E}{d D}\right|_{D_{m}}$ versus $N$. This has been plotted in Fig 14 which shows a linear behavior of $\ln (y)$ versus $\ln (N)$. The exponent for this behavior is $\left|\frac{d E}{d D}\right|_{D_{m}} \sim$ $N^{0.46}$. This results justify that the RG implementation of entanglement truly capture the critical behavior of the model at $D=1$. It should be emphasized this exponent is directly related to the correlation length exponent, $\nu$, close to the critical point. It has been shown in Ref. 27] that $\left|\frac{d E}{d D}\right|_{D_{c}} \sim N^{1 / \nu}$ and $D_{m}=D_{c}+N^{-1 / \nu}$.

To study the scaling behavior of the entanglement en-

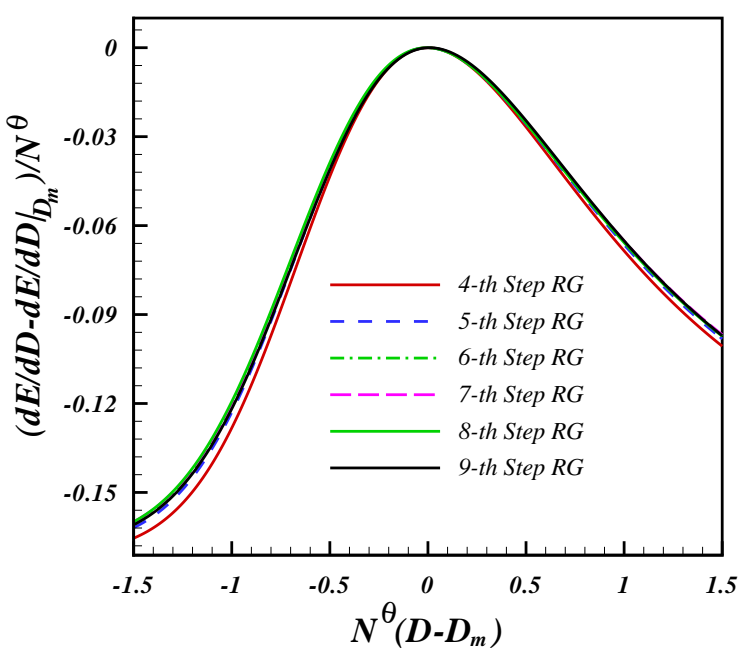

FIG. 15: (color online) The finite size scaling is performed via the RG treatment for the power-law scaling. Each curve corresponds to a definite size of the system, i.e $N=3^{n+1}$. The exponent $\theta$ is ascribed to the correlation length critical exponent $\nu$ via $\theta=1 / \nu$

tropy around the critical point, we perform finite scaling analysis. Since the minimum value of derivative of entanglement entropy scales power-law. According to the scaling ansatz, the rescaled derivative of entanglement entropy around its minimum value, $D_{m}$, is just a function of rescaled driving parameter like:

$$
\frac{\frac{d E}{d D}-\left.\frac{d E}{d D}\right|_{D_{m}}}{N^{\theta}}=F\left[N^{\theta}\left(D-D_{m}\right)\right]
$$

where, $F(x)$ is a universal function that does not deponed on the system size, and the exponent $\theta$ is just the inverse of the critical exponent $\nu$, i.e $\theta=1 / \nu$. The manifestation of the finite size scaling is shown in Fig[15, It is clear that the different curves which are resemblance of various system sizes collapse to a single universal curve. It must be noticed that the $n-t h$ RG iteration describes a system with $3^{n+1}$ sites which is effectively represented by a three site model through the RG treatment.

\section{SUMMARY AND CONCLUSIONS}

We have applied the quantum RG approximation to obtain the phase diagram, staggered magnetization, chiral order and the entanglement properties of Ising model with DM interaction. Tunning the DM interaction dictated the system to fall into different phases, i.e antiferromagnetic phase with nonzero staggered magnetization (as order parameter) and chiral one with vanishing order parameter. The critical point of the phase transition is at $D_{c}=1$ where the quantum fluctuations have dominant 
effect which arises from the DM interaction and eventually destroy the order in the AF phase. Although the DM interaction drives the spins to leave their ordering in the z-direction, i.e staggered magnetization, a chiral saturated phase has been arisen, Fig, 2

The numerical exact diagonalization which has been done on finite sizes $(N=12,16,18,20)$ justifies the results of QRG. We have obtained a fairly well agreement in the ground state energy of QRG approach with exact diagonalization method. The divergence of $z$-component structure factor at momentum $k=\pi$ when $N \rightarrow \infty$ is the signature of antiferromagnetic long range order for $D<D_{c}$. The value of critical point which is read from exact diagonalization is $D_{c}^{\text {numeric }} \simeq=0.8$ which is 20 percent different from what QRG gives, $D_{c}^{Q R G}=1$. However, we claim that the QRG result for $D_{c}$ should be more reliable as will be discussed below in connection with anisotropic Heisenberg (XXZ) model. Moreover, the size dependence of the critical point $\left(D_{c}\right)$ is strong which demands the numerics on larger sizes and also a scaling analysis.

Besides, the entanglement entropy of the model at different RG iterations was analyzed. As the long wavelength behavior of the model is reached via the increasing of the RG iterations, the entanglement entropy develops two distinct behavior proportional to two different existing phases of the model. However nonanalytic behavior close to the critical point of the model manifests itself via the analysis of the first derivative of the entanglement entropy. The divergence of the first derivative of the entanglement entropy becomes more pronounced as long as the size of the system becomes large in RG treatment. Critical point is touched by an exponent which appears as the inverse of the critical exponent which shows the divergence of the correlation length. Moreover, it is found that as the critical point is touched from the gapped phase a drastic change in the ground state occurs which manifests itself in the evolution of the derivative of entanglement (see Fig 12). Such variation in the ground state structure also appears in the $X X Z$ model. Finally finite size scaling reveals the critical properties of the model is mirrored via the nonanalytic behavior of the entanglement.

The one dimensional Ising model with DM interaction (Eq.(1)) is mapped to the $X X Z$ chain via a nonlocal canonical transformation ${ }^{36,37}$,

$$
\begin{gathered}
U=\sum_{j=1}^{N} \alpha_{j} \sigma_{j}^{z} \quad, \quad \alpha_{j}=\sum_{m=1}^{j-1} m \tan ^{-1}(D), \\
\tilde{\sigma}_{j}^{ \pm}=e^{-i U} \sigma_{j}^{ \pm} e^{i U}, \quad \tilde{\sigma}_{j}^{z}=\sigma^{z} \\
\tilde{H}=e^{-i U} H e^{i U},
\end{gathered}
$$

which gives

$$
\tilde{H} \sim \sum_{i}\left(\tilde{\sigma}_{i}^{x} \tilde{\sigma}_{i+1}^{x}+\tilde{\sigma}_{i}^{y} \tilde{\sigma}_{i+1}^{y}+\left(\frac{1}{D}\right) \tilde{\sigma}_{i}^{z} \tilde{\sigma}_{i+1}^{z}\right) .
$$

This transformation tells that the system is in the spin-fluid phase for $D>1$ in terms of transformed spins. While, the model represents the antiferromagnetic (Néel) phase for $D<1$. The spin-fluid phase $(D>1)$ of the transformed Hamiltonian $(\tilde{H})$ can be characterized by a string order parameter ${ }^{38}$. At the same time the saturated chiral phase $(D>1)$ of the original Hamiltonian (Eq.(1)) is represented by the chiral order. It can be concluded that the chiral order with nonlocal spins is similar to the string order parameter. Therefore, it is suggested that the $X X Z$ model has chiral order which is constructed with nonlocal spins. It will be instructive to calculate the chiral order with nonlocal spins for $X X Z$ model as a hidden order of the spin-fluid phase.

\section{Acknowledgments}

The authors would like to thank Prof. M. R. H. Khajehpour for careful reading of the manuscript and fruitful discussions. We would like also acknowledge J. Abouie for useful discussions. This work was supported in part by the Center of Excellence in Complex Systems and Condensed Matter (www.cscm.ir).

\section{APPENDIX A: THE BLOCK HAMILTONIAN OF THREE SITES, ITS EIGENVECTORS AND EIGENVALUES}

We have considered the three-site block (Fig.(1D) with the following Hamiltonian

$$
\begin{aligned}
h_{I}^{B}=\frac{J}{4}[ & \left(\sigma_{1, I}^{z} \sigma_{2, I}^{z}+\sigma_{2, I}^{z} \sigma_{3, I}^{z}\right)+D\left(\sigma_{1, I}^{x} \sigma_{2, I}^{y}\right. \\
& \left.\left.-\sigma_{1, I}^{y} \sigma_{2, I}^{x}+\sigma_{2, I}^{x} \sigma_{3, I}^{y}-\sigma_{2, I}^{y} \sigma_{3, I}^{x}\right)\right] .
\end{aligned}
$$

The inter-block $\left(H^{B B}\right)$ and intra-block $\left(H^{B}\right)$ Hamiltonian for the three sites decomposition are

$$
\begin{aligned}
H^{B} & =\frac{J}{4} \sum_{i=1}^{N / 3}\left[\sigma_{1, I}^{z} \sigma_{2, I}^{z}+\sigma_{2, I}^{z} \sigma_{3, I}^{z}\right. \\
& \left.+D\left(\sigma_{1, I}^{x} \sigma_{2, I}^{y}-\sigma_{1, I}^{y} \sigma_{2, I}^{x}+\sigma_{2, I}^{x} \sigma_{3, I}^{y}-\sigma_{2, I}^{y} \sigma_{3, I}^{x}\right)\right], \\
H^{B B} & =\frac{J}{4} \sum_{I=1}^{N / 3}\left[\sigma_{3, I}^{z} \sigma_{1, I+1}^{z}+D\left(\sigma_{3, I}^{x} \sigma_{1, I+1}^{y}-\sigma_{3, I}^{y} \sigma_{1, I+1}^{x}\right)\right],
\end{aligned}
$$

where $\sigma_{j, I}^{\alpha}$ refers to the $\alpha$-component of the Pauli matrix at site $j$ of the block labeled by $I$. The exact treatment of this Hamiltonian leads to four distinct eigenvalues which are doubly degenerate. The ground, first, second and third excited state energies have the following expressions in terms of the coupling constants. 


$$
\begin{aligned}
\left|\psi_{0}\right\rangle & \left.=\frac{1}{\sqrt{2 q(1+q)}}[2 D|\downarrow \uparrow \uparrow \uparrow+i(1+q)| \uparrow \downarrow \uparrow\rangle-2 D|\uparrow \uparrow \downarrow\rangle\right], \\
\left|\psi_{0}^{\prime}\right\rangle & =\frac{1}{\sqrt{2 q(1+q)}}[2 D|\downarrow \downarrow \uparrow\rangle+i(1+q)|\downarrow \uparrow \downarrow\rangle-2 D|\uparrow \downarrow \downarrow\rangle], \\
e_{0} & =-\frac{J}{4}(1+q)
\end{aligned}
$$$$
\left.\left|\psi_{1}\right\rangle=\frac{1}{\sqrt{2 q(q-1)}}[2 D|\downarrow \uparrow \uparrow\rangle\rangle-i(q-1)|\uparrow \downarrow \uparrow\rangle-2 D|\uparrow \uparrow \downarrow\rangle\right],
$$$$
\left|\psi_{1}^{\prime}\right\rangle=\frac{1}{\sqrt{2 q(q-1)}}[2 D|\downarrow \downarrow \uparrow\rangle-i(q-1)|\downarrow \uparrow \downarrow\rangle-2 D|\uparrow \downarrow \downarrow\rangle],
$$$$
e_{1}=-\frac{J}{4}(1-q) \text {, }
$$$$
\left|\psi_{2}\right\rangle=\frac{1}{\sqrt{2}}(|\uparrow \uparrow \downarrow\rangle+|\downarrow \uparrow \uparrow\rangle), \quad\left|\psi_{2}^{\prime}\right\rangle=\frac{1}{\sqrt{2}}(|\downarrow \downarrow \uparrow\rangle+|\uparrow \downarrow \downarrow\rangle),
$$$$
e_{2}=0 \text {, }
$$$$
\left|\psi_{3}\right\rangle=|\uparrow \uparrow \uparrow\rangle, \quad\left|\psi_{3}^{\prime}\right\rangle=|\downarrow \downarrow \downarrow\rangle,
$$$$
e_{3}=\frac{J}{2}
$$

function at each iteration of RG is connected to its value after an RG iteration. This will be continued to reach a controllable fixed point where we can obtain the value of the correlation function. The staggered magnetization in $\alpha$ direction can be written

$$
S_{M}=\frac{1}{N} \sum_{i}^{N}\left\langle O\left|\frac{(-1)^{i}}{2} \sigma_{i}^{\alpha}\right| O\right\rangle,
$$

where $\sigma_{i}^{\alpha}$ is the Pauli matrix in the $i$ th site and $|O\rangle$ is the ground state of chain. The ground state of the renormalized chain is related to the ground state of the original one by the transformation, $P_{0}\left|O^{\prime}\right\rangle=|O\rangle$.

$$
S_{M}=\frac{1}{N} \sum_{i}^{N}\left\langle O^{\prime}\left|P_{0}\left(\frac{(-1)^{i}}{2} \sigma_{i}^{\alpha}\right) P_{0}\right| O^{\prime}\right\rangle .
$$

This leads to the staggered configuration in the renormalized chain. The staggered magnetization in $z$ direction is (A2)bbtained

where $q$ is $q=\sqrt{1+8 D^{2}}$.

$|\uparrow\rangle$ and $|\downarrow\rangle$ are the eigenstates of $\sigma^{z}$. The projection operator is

$$
P_{0}=|\Uparrow\rangle\left\langle\psi_{0}|+| \Downarrow\right\rangle\left\langle\psi_{0}^{\prime}\right| .
$$

The Pauli matrices in the effective Hilbert space have the following transformations

$$
\begin{aligned}
& P_{0}^{I} \sigma_{1, I}^{x} P_{0}^{I}=\frac{2 D}{q}{\sigma^{\prime}}_{I}^{y}, \quad P_{0}^{I} \sigma_{2, I}^{x} P_{0}^{I}=\frac{4 D^{2}}{q(q+1)}{\sigma^{\prime}}_{I}^{x}, \\
& P_{0}^{I} \sigma_{3, I}^{x} P_{0}^{I}=-\frac{2 D}{q} \sigma_{I}^{\prime y} \quad, P_{0}^{I} \sigma_{1, I}^{y} P_{0}^{I}=-\frac{2 D}{q}{\sigma_{I}^{\prime}}_{I}^{x}, \\
& P_{0}^{I} \sigma_{2, I}^{y} P_{0}^{I}=\frac{4 D^{2}}{q(q+1)} \sigma_{I}^{\prime y}, \quad P_{0}^{I} \sigma_{3, I}^{y} P_{0}^{I}=\frac{2 D}{q}{\sigma^{\prime}}_{I}^{x} \text {, } \\
& P_{0}^{I} \sigma_{1, I}^{z} P_{0}^{I}=\frac{1+q}{2 q}{\sigma^{\prime}}_{I}^{z} \quad, P_{0}^{I} \sigma_{2, I}^{z} P_{0}^{I}=-\frac{1}{q}{\sigma^{\prime}}_{I}^{z}, \\
& P_{0}^{I} \sigma_{3, I}^{z} P_{0}^{I}=\frac{1+q}{2 q} \sigma_{I}^{\prime z} \text {. }
\end{aligned}
$$

\section{APPENDIX B: ORDER PARAMETER AND CHIRAL ORDER}

\section{Staggered magnetization}

Generally, any correlation function can be calculated in the QRG scheme. In this approach, the correlation

$$
\begin{aligned}
S_{M}^{0} & =\frac{1}{N} \sum_{i=1}^{N}\left\langle 0\left|\frac{(-1)^{i}}{2} \sigma_{i}^{z}\right| 0\right\rangle \\
& =\frac{1}{6} \frac{1}{\frac{N}{3}} \sum_{I=1}^{N / 3}\left[\left\langle 0^{\prime}\left|P_{0}^{I}\left(-\sigma_{1, I}^{z}+\sigma_{2, I}^{z}-\sigma_{3, I}^{z}\right) P_{0}^{I}\right| 0^{\prime}\right\rangle\right. \\
& \left.-\left\langle 0^{\prime}\left|P_{0}^{I+1}\left(-\sigma_{1, I+1}^{z}+\sigma_{2, I+1}^{z}-\sigma_{3, I+1}^{z}\right) P_{0}^{I+1}\right| 0^{\prime}\right\rangle\right] \\
& =-\left(\frac{2+q}{3 q}\right) \frac{1}{\frac{N}{3}} \sum_{I=1}^{N / 3}\left\langle 0^{\prime}\left|\frac{(-1)^{I}}{2} \sigma_{I}^{z}\right| 0^{\prime}\right\rangle=-\frac{\gamma^{0}}{3} S_{M}^{1},(\mathrm{~B} 2)
\end{aligned}
$$

where $S_{M}^{(n)}$ is the staggered magnetization at the $n$th step of QRG and $\gamma^{(0)}$ is defined by $\gamma^{0}=(2+q) / q$.

This process will be iterated many times by replacing $\gamma^{(0)}$ with $\gamma^{(n)}$. The expression for $\gamma^{(n)}$ is similar to $\gamma^{(0)}$ where the coupling constants should be replaced by the renormalized ones at the corresponding $\mathrm{RG}$ iteration $(n)$. The result of this calculation has been presented in Fig.(3).

\section{Chiral Order}




$$
\begin{aligned}
C_{h} & =\frac{1}{N} \sum_{i=1}^{N} \frac{1}{4}\left\langle 0\left|\left(\sigma_{i}^{x} \sigma_{i+1}^{y}-\sigma_{i}^{y} \sigma_{i+1}^{x}\right)\right| 0\right\rangle \\
& =\frac{1}{12} \frac{1}{\frac{N}{3}} \sum_{I=1}^{N / 3}\left[\left\langle 0^{\prime}\left|P_{0}\left(\sigma_{3, I}^{x} \sigma_{1, I+1}^{y}-\sigma_{3, I}^{y} \sigma_{1, I+1}^{x}\right) P_{0}\right| 0^{\prime}\right\rangle+\left\langle 0^{\prime}\left|P_{0}^{I}\left(\left(\sigma_{1, I}^{x} \sigma_{2, I}^{y}-\sigma_{1, I}^{y} \sigma_{2, I}^{x}\right)+\left(\sigma_{2, I}^{x} \sigma_{3, I}^{y}-\sigma_{2, I}^{y} \sigma_{3, I}^{x}\right)\right) P_{0}^{I}\right| 0^{\prime}\right\rangle\right] \\
& =\frac{1}{12} \frac{32 D^{3}}{q^{2}(1+q)}+\frac{1}{3} \frac{1}{\frac{N}{3}}\left(\frac{2 D}{q}\right)^{2} \sum_{I=1}^{N / 3} \frac{1}{4}\left\langle 0^{\prime}\left|\left(\sigma_{I}^{x} \sigma_{I+1}^{y}-\sigma_{I}^{y} \sigma_{I+1}^{x}\right)\right| 0^{\prime}\right\rangle=C^{0}+\frac{\Upsilon^{0}}{3} C_{h}^{1}, C^{0}=\frac{1}{3} \frac{32 D^{3}}{q^{2}(1+q)}, \quad \Upsilon^{0}=\left(\frac{2 D}{q}\right)^{2}
\end{aligned}
$$

At the last step we use the following transformation, $\sigma_{i}^{z} \rightarrow-\sigma_{i}^{z}, \sigma_{i}^{y} \rightarrow-\sigma_{i}^{y}$.

\section{APPENDIX C: CLASSICAL APPROXIMATION}

In the classical approximation the spins are considered as classical vectors

$$
\sigma_{i}^{x}=\cos (i \varphi) \sin \theta, \sigma_{i}^{y}=\sin (i \varphi) \sin \theta, \sigma_{i}^{z}=\cos \theta,
$$

where $\varphi$ is the azimuthal angle measured from the $x$ axis in the $x y$-plane and $\theta$ is the polar angle measured down from the $z$-axis. The classical energy per site for IDM Hamiltonian (Eq.(1)) is:

$$
\frac{E_{c l}}{N}=\frac{J}{4}\left(\cos ^{2} \theta+D \sin ^{2} \theta \sin \varphi\right) .
$$

The minimization of classical energy with respect to the angles $\varphi$ and $\theta$ shows that there are two different regions. (I) $D>1$, the minimum of energy is obtained by $\theta=\frac{\pi}{2}$ and $\varphi=\arcsin \left(\frac{1}{D}\right)$ which show the spins do not have a projection on the $z$-axis and have the helical structure (see Fig (4) in the $x y$ plain. In this region the minimum classical energy is

$$
\frac{E_{c l}^{I}}{N}=\frac{J}{4}
$$

(II) $D<1$, the energy is minimized by $\phi=\frac{\pi}{2}$ and arbitrary $\theta$ which corresponds to the configuration with nonzero value of spins projection on $z$-axis and helical structure of spins projection in the $x y$-plain where the angle between spins are $\frac{\pi}{2}$. In this region the minimum classical energy is

$$
\frac{E_{c l}^{I I}}{N}=\frac{J}{4}\left(\cos ^{2} \theta+D \sin ^{2} \theta\right) .
$$

One can see from Eq.(C1) and Eq. (C2) that the transition between phase (I) and (II) takes place at $D=1$.

\section{REFERENCES}

1 J. Bardeen, L. N. Cooper and J. R. Schrieffer, Phys. Rev. 108, 1175 (1957).

2 R. B. Laughlin, Phys. Rev. Lett. 50, 1395 (1983).

3 S. Sachdev, Quantum phase transition, Cambridge Uni. Press (1999).

4 M. Vojta, Rep. Prof. Phys. 66, 2069 (2003) and references therein.

5 J. Preskill, J. Mod. Opt 47, 127 (2000).

${ }^{6}$ I. Dzyaloshinskii, J. Phys. Chem. Solids 4, 241 (1958).

7 T. Moriya, Phys. Rev 120, 91 (1960).

8 D. C. Dender, D. Davidovic, D. H. Reich, C. Broholm, K. Lefmann, and G. Aeppli, Phys. Rev. B 53, 2583 (1996).

9 D. C. Dender, P. R. Hammar,D. H. Reich, C. Broholm , and G. Aeppli Phys. Rev. Lett 79, 1750 (1997).

10 M. Kohgi, K. Iwasa, J. M. Mignot, B. Fak, P. Gegenwart, M. Lang, A. Ochiai, H. Aoki, and T. Suzuki, Phys. Rev. Lett 86, 2439 (2001).

11 P. Fulde B. Schmidt, and P. Thalmeier, Europhys. Lett 31, 323 (1995).
12 M. Osikawa, K. Ueda, H. Aoki, A. Ochiai and M. Kohgi, J. Phys. Soc. Jpn 68, 3181 (1999); H. Shiba, K. Udea, and O. Sakai, J. Phys. Soc. Jpn 69, 1493 (2000)

13 I. Tsukada, J. T. Takeya, T. Masuda and K. Uchinokura, Phys. Rev. Lett 87, 127203 (2001)

14 b. Grande and Hk. Müller-Buschbaum, Z. Anorg. Allg. Chem 417, 68 (1975).

15 M. Greven, R. J. Birgeneau, Y. Endoh, M. A. Kastner, M. Matsuda, and G. Shirane, Z. Phys. B 96, 465 (1995).

16 T. Yildirim, A. B. Harris, A. Aharony and O. EntinWohlman, Phys. Rev. B. 52, 10239 (1995).

17 K. Katsumata, M. Hagiwara,Z. Honda, J. Satooka, A. Aharoy, R. J. Birgeneau, F. C. Chou, O. E. Wohlman, A. B. Harris,M. A. Kastner, Y. J. Kim, and Y. S. Lee, Europhys. Rev. Lett 54, 508(2001).

18 M. A. Kastnetr, R. J. Birgeneau, G. Shirane and Y. Endoh, Rev. Mod. Phys 70, 897 (1998).

19 M. Fiebig, J. Phys. D: Appl. Phys 38, R123 (2005).

${ }^{20}$ T. Kimura and T. Goto, H. Shintani, K. Ishizaka, T. 
Arima, and Y. Tokura Nature 426, 55 (2003).

21 N. Hur, S. Park, P. A. Sharma. J. S. Ahn, S. Guha, and S.-W. Cheong Nature 429, 392 (2004).

22 T. Kimura, G. Lawes, and A. P. Ramirez, Phys. Rev. Lett 94, 137201 (2005).

${ }^{23}$ G. Lawes, A. B. Harris, T. Kimura, N. Rogado, R. J. Cava, A. Aharony, O. Entin-Wohlman, T. Yildrim, M. Kenzelmann, C. Broholm, and A. P. Ramirez, Phys. Rev. Lett 95, 087205 (2005).

${ }^{24}$ M. Kenzelmann, A. B. Harris, S. Jonas, C. Broholm, J. Schefer, S. B. Kim, C. L. Zhang, S.-W. Cheong, O. P. Vajk, and J. W. Lynn, Phys. Rev. Lett 95, 087206 (2005).

25 I. A. Sergienko and E. Dagotto, Phys. Rev. B 73, 094434 (2006).

26 M. Kargarian, R. Jafari, A. Langari, Phys. Rev. A 76, 060304(R) (2007).

27 M. Kargarian, R. Jafari, A. Langari, Phys. Rev. A 77, 032346 (2008).

28 M. A. Martin-Delgado and G. Sierra, Int. J. Mod, Phys. A 11, 3145 (1996).

29 G. Sierra and M. A. Martin Delgado, in Strongly Correlated
Magnetic and Superconducting Systems, Lecture Notes in Physics Vo1. 478 (springer, Berlin, 1997).

30 A. Langari, Phys. Rev. B 58, 14467 (1998); 69, 100402(R) (2004).

31 R. Jafari, A. Langari, Phys. Rev. B 76, 014412 (2007); Physica A 364, 213 (2006).

${ }^{32}$ H. Kawamura, Phys. Rev. B 38, 4916 (1988).

33 N. Goldenfeld, Lectures on phase transition and the Renormalization Group, Addison-Wesley Publlishing group (1992).

34 A. Osterloh, luigi Amico, G. Falci, and Rosario Fazio, Nature 416, 608 (2002).

${ }^{35}$ G. Vidal, J. I. Latorre, E. Rico, and A. Kitaev, Phys. Rev. Lett 90, 227902 (2003).

36 D. N. Aristov, S. V. Maleyev, Phys. Rev. B 62, R751 (2000).

37 F. C. Alcaraz and W. F. Wreszinski, J. Stat. Phys. 58, 45 (1990).

38 K. Hida, Phys. Rev. B 45, 2207, (1992). 